\title{
Optimization of Broadband Y-Junction Splitters in Fused Silica by Femtosecond Laser Writing
}

\author{
Vítor A. Amorim, João M. Maia, D. Alexandre, and P. V. S. Marques
}

\begin{abstract}
Optical Y-junction power splitters owe their inherent broadband spectral behavior to their design. However, depending on the fabrication technique employed, asymmetries in the junction might arise, perturbing its performance; this is the case in femtosecond laser written Y-junctions where one arm is typically written over the top of the other. In this letter, the spectral behavior of Y-junctions fabricated in fused silica by the femtosecond laser direct writing technique was analyzed and optimized for the first time, to the best of our knowledge. The junction arms output power balance as well as the corresponding spectral flatness between 1300 and $1600 \mathrm{~nm}$ is substantially increased by the implementation of an initial separation between the arms at the junction diverging point, enabling the manufacturing of balanced broadband Y-junctions.
\end{abstract}

Index Terms-Integrated optics, optical device fabrication, laser materials-processing applications, femtosecond laser, optical waveguide components, optical beam splitting, Y-junctions.

\section{INTRODUCTION}

$\mathbf{Y}$ -JUNCTIONS are devices defined by the branching of a single-mode waveguide into two equal single-mode waveguides. The main characteristic of this device, that differentiate it from other power splitters such as multimode interference devices (MMIs) [1] and directional couplers [2], is its inherent achromatic behavior [3], which allows $3 \mathrm{~dB}$ power splitting over a large range of wavelengths. They can also be combined together in binary tree structures [4], allowing a single input to be equally split into multiple outputs or vice-versa.

Y-junctions are often used as optical power splitters or optical power combiners in modulators and switches [5], made by joining two Y-structures and introducing a phase shifter into one of the arms [6].

Manuscript received January 31, 2017; revised February 15, 2017; accepted February 23, 2017. Date of publication March 1, 2017; date of current version March 14, 2017. This work was supported in part by the Calouste Gulbenkian Foundation through the Stimulus to Scientific Research Program under Grant 141773 and in part by the North Portugal Regional Operational Programme 2020, under the PORTUGAL 2020 Partnership Agreement, and the European Regional Development Fund through the Project NanoSTIMA: Macro-to-Nano Human Sensing: Toward Integrated Multimodal Health Monitoring and Analytics/NORTE-01-0145-FEDER-000016.

V. A. Amorim, J. M. Maia, and P. V. S. Marques are with the Center for Applied Photonics, INESC TEC, 4169-007 Porto, Portugal, and also with the Department of Physics and Astronomy, University of Porto, 4169-007, Porto, Portugal (e-mail: vitor.a.amorim@inesctec.pt; joao.m.maia@inesctec.pt; psmarque@fc.up.pt).

D. Alexandre is with the Center for Applied Photonics, INESC TEC, 4169007 Porto, Portugal, and also with the Department of Physics, University of Trás-os-Montes e Alto Douro, 5001-801 Vila Real, Portugal (e-mail: daniel@utad.pt).

Color versions of one or more of the figures in this letter are available online at http://ieeexplore.ieee.org.

Digital Object Identifier 10.1109/LPT.2017.2675858
High performance Y-junctions (low insertion losses, high bandwidth, and high power uniformity between branches) have already been demonstrated using Planar Lightwave Circuit (PLC) technology [7], [8]. While PLC technology offers significant advantages in scalability, cost, and circuit compactness, femtosecond laser direct writing techniques can offer prototyping flexibility and real-time optimizations that are not present in PLC fabrication methods. Furthermore, it allows easy monolithic integration of optofluidic devices for bioanalysis [9] due to the technique's inherent high spatial resolution and 3D capabilities. However, in laser direct writing processes, symmetry is difficult to achieve when there is an overlap of the laser scanning lines, which is the case on the splitting area of the Y-junction. This has been the case on fabrication methods that depends on linear photosensitivity due to depletion of dissolved molecular hydrogen [10] and also in nonlinear processes like the ones that are employed on this letter. The fabrication of Y-junctions with type 1 waveguides using the femtosecond laser direct writing technique has already been reported in several materials [11]-[14]. Most advances in this area were made by Liu et al. [15], by the use of a multi-scan technique to decrease both insertion loss and polarization dependent loss, demonstrating $1 \times \mathrm{N}$ splitters in fused silica which show relatively good power splitting balance at $1550 \mathrm{~nm}$ with a large insertion loss $(6.72 \mathrm{~dB}$ for $25 \mathrm{~mm}$ long $1 \times 2$ splitters) due to the waveguides high propagation loss, and Sohn et al, which used micromachined U-grooves for passive fiber alignment, demonstrating $1 \times 2$ [16] and $1 \times 4$ [17] splitters fabricated again with high propagation loss waveguides $(0.86 \mathrm{~dB} / \mathrm{cm})$ at $1550 \mathrm{~nm}$. Given this, more attention should be invested in the fabrication of high performance devices, in particular in what concerns the optimization of the Y-junction design for power balance in a broad wavelength range as well as spectral flatness which has never been explored to the authors' best knowledge.

In this letter we optimize the design of Y-junction splitters, fabricated by the femtosecond laser direct writing technique, to enable balanced power splitting over broad wavelength ranges with improved spectral flatness. The impact of arm separation, at the junction diverging point, in the spectral response was studied between $1300 \mathrm{~nm}$ and $1600 \mathrm{~nm}$ and the best fabrication parameters were found.

\section{EXPERIMENTAL PROCEDURE}

The femtosecond laser system used for the fabrication of the Y-junctions was an Amplitude Systemes Satsuma HP fiber amplified laser. The second harmonic beam at $\lambda=515 \mathrm{~nm}$, 


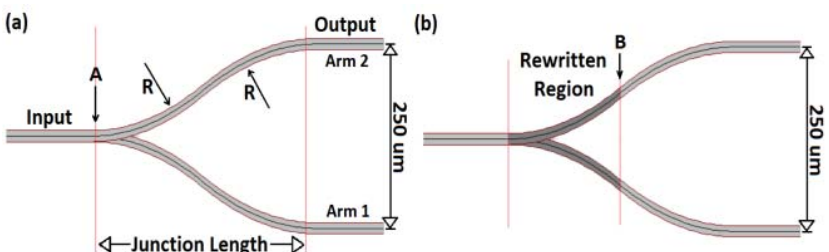

(c)

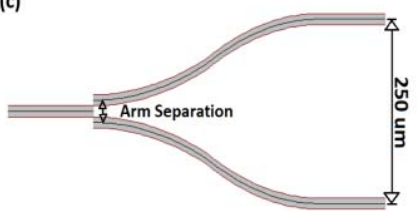

Fig. 1. Y-junction design for the first test (a), with branching at the same spot, second test (b), with multiple arm writing, and third test (c), with an initial arm separation.

with an approximate $250 \mathrm{fs}$ pulse duration and a repetition rate of $500 \mathrm{kHz}$, was focused inside a fused silica substrate $50 \mu \mathrm{m}$ below the surface with a 0.55 numerical aperture aspherical lens [18]. To fabricate low loss optical waveguides a calibration was made, with optimal writing conditions being obtained with laser pulses of $250 \mathrm{~nJ}$ at a sample scanning speed of $400 \mu \mathrm{m} / \mathrm{s}$. Also, a linear writing beam polarization aligned with the scanning direction was used, since lower propagation loss and birefringence are obtained [19]. Writing direction was also optimized, with all devices being manufactured always in the same direction to avoid Quill effect [20]. These exposure conditions yielded waveguides with total insertion losses of $1.1 \mathrm{~dB}$, for $2.5 \mathrm{~cm}$ long waveguides, with a mode field diameter of $12.3 \mu \mathrm{m} \times 7.1 \mu \mathrm{m}$, resulting in coupling losses of $0.37 \mathrm{~dB}$ per facet, when butt-coupled to a SMF28 fiber, and propagation losses of $0.14 \mathrm{~dB} / \mathrm{cm}$ at $1550 \mathrm{~nm}$, which are in line with the best results in literature [21], [22]. To minimize bending loss the radius of arc shaped S-bends was also optimized; a curvature radius of $80 \mathrm{~mm}$ and $125 \mu \mathrm{m}$ lateral offset were employed, resulting in a $250 \mu \mathrm{m}$ separation between outputs and a $6.2 \mathrm{~mm}$ long junction.

\section{EXPERIMENTAL RESULTS AND DISCUSSION}

To study and optimize the Y-junctions fabrication parameters the conventional configuration of Fig. 1(a) was initially tested with the branching starting completely overlapped at the same spot (point A of Fig. 1(a)). This was accomplished by writing the input waveguide followed by arm 1 and then returning to point A to write arm 2. Ten Y-junctions employing this design were fabricated, with a mean power splitting of 44.9/55.1\% being obtained for arm 1 and 2 respectively, at $1550 \mathrm{~nm}$, with a standard deviation of $1.2 \%$. This evidences the existence of a consistent asymmetry in the fabricated junctions, since more power is flowing into arm 2 (the last arm written), with a $\sim 5 \%$ deviation from the ideal behavior (50\%). By looking in more detail into the overlapped zone, Fig. 2(b), the deviation can be explained since the last arm written is visibly more defined. This effect is probably due to the fact that refractive index saturation was not reached during the first laser scan in the fabrication of the first arm, resulting in an

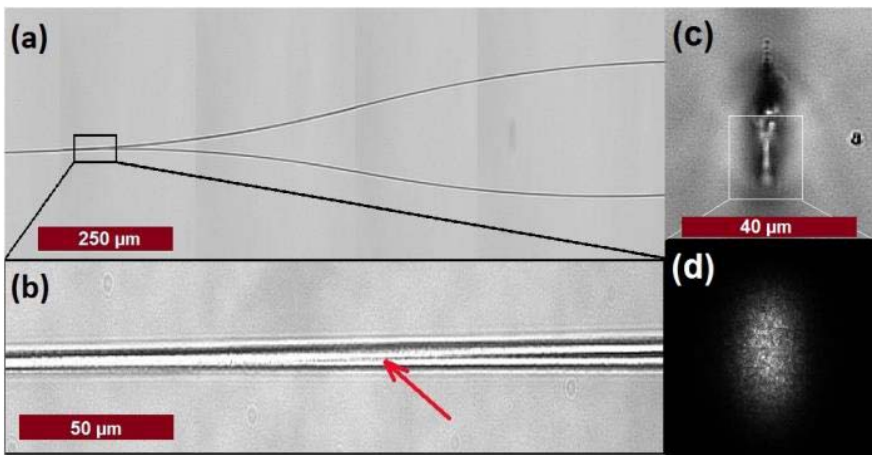

Fig. 2. (a) Optical microscope images of the top view of the Y-junctions fabricated following the design of Fig. 1(a). (b) Zoomed image showing the branching of both arms. (c) Cross sectional microscope image and (d) $1550 \mathrm{~nm}$ mode profile of the waveguide written.

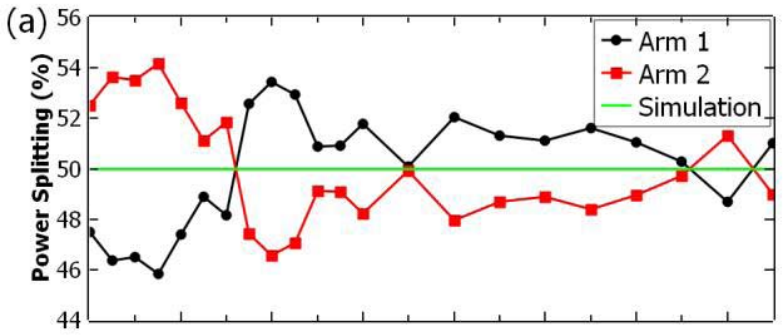

(b)

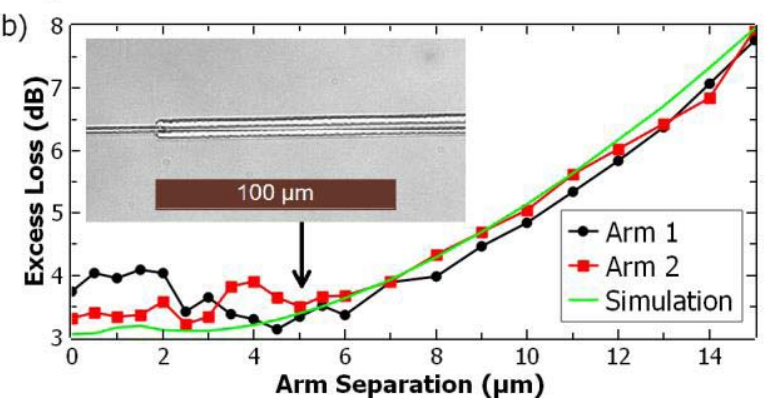

Fig. 3. Analysis of Y-junctions, at $1550 \mathrm{~nm}$, fabricated with different arm separation at the diverging point. Power splitting (a) and excess loss (b) is plotted as a function of arm separation. The inset shows the junction of a splitter fabricated with a separation of $5 \mu \mathrm{m}$ between arms.

asymmetric index distribution in the branching zone of the Y-junction.

To correct this problem one might think of saturating the refractive index change in that region by performing multiple writings. This was tested by rewriting the arms (up to 10 times for each arm) sequentially from the diverging point up to half of the S-bend (point B in Fig. 1(b)), but it was observed that the junction spectral behavior becomes increasingly irregular with an increasing number of scans. The probable cause relates to the increasing complexity of the refractive index of the modified region, since the energy distribution of the writing laser focus is altered in each pass.

A better solution was found separating the Y-junctions arms, as seen in Fig. 1(c). The idea was to find the minimum separation that eliminates the junction asymmetry, by reducing the beam overlap. For this study several Y-junctions were fabricated with arm separations ranging from $0 \mu \mathrm{m}$ (fully overlapped conventional Y-junction) to $15 \mu \mathrm{m}$. The fabricated devices were characterized at $1550 \mathrm{~nm}$ and the experimental 

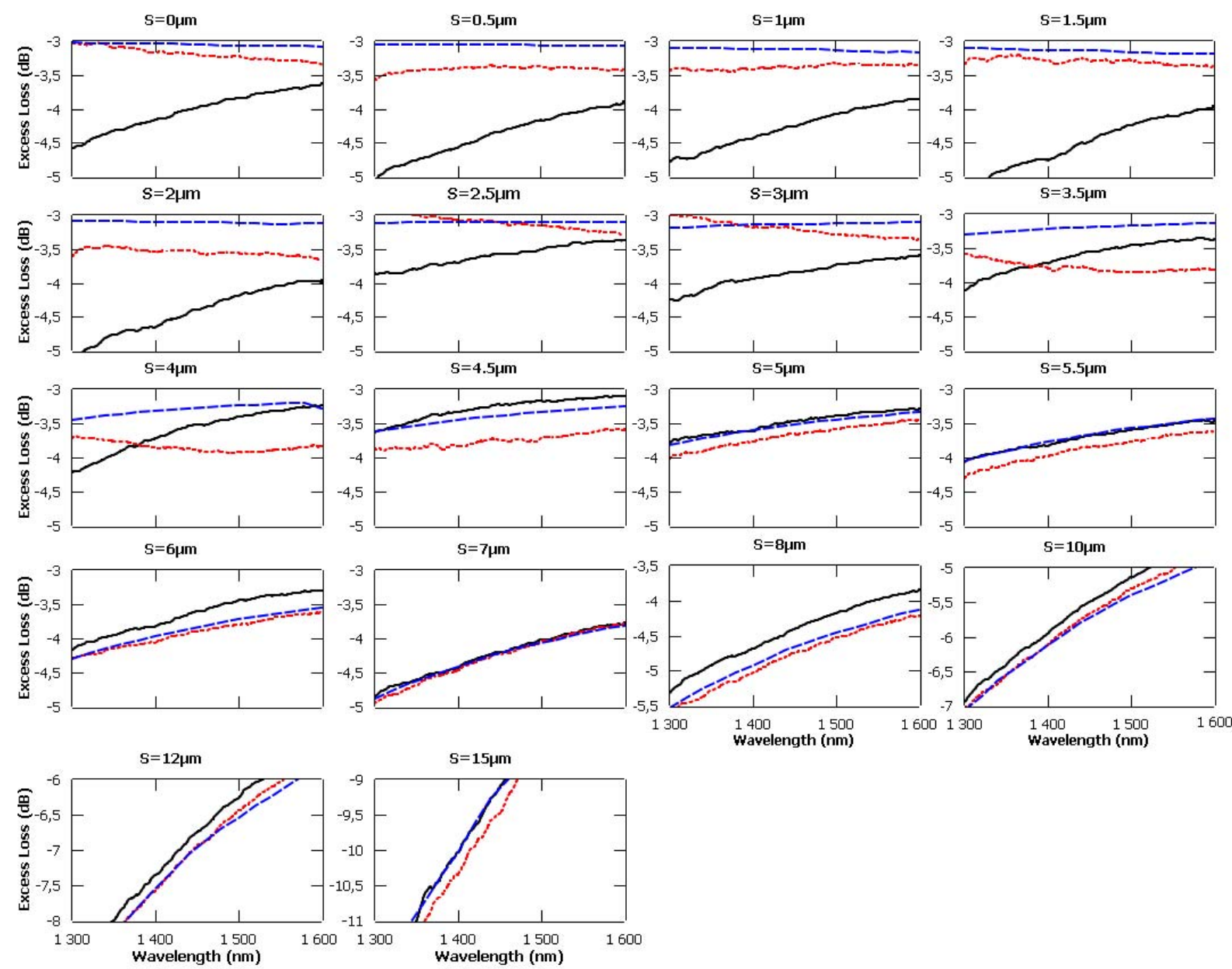

Fig. 4. Spectral behavior of Y-junctions fabricated with different arm separations at the diverging point. It should be noted that, although the vertical scale is identical for all individual graphics, the vertical scale changes for separations larger than $7 \mu \mathrm{m}$ due to increasing light loss. Solid Line-Arm 1; Doted Line-Arm 2; Dashed Line-Simulation results.

results are presented in Fig. 3. The simulation results were obtained with Rsoft BeamPROP using a 2D model with a 2.03 $\mu \mathrm{m}$ waveguide cross section and $9.5 \times 10^{-3}$ refractive index difference between the core and the cladding sections. The waveguide simulation parameters were chosen by comparing Y-junction simulations with experimental data (for a separation larger than $5 \mu \mathrm{m}$ ) as a function of wavelength for different arm separations. Several waveguide parameters yielding different mode field diameters were used for a coarse adjustment, while different waveguide parameters yielding the same mode field diameter were used to make a fine adjustment. The results show that the power splitting becomes closer to the simulated $3 \mathrm{~dB}$ splitting ratio with increasing arm separation (Fig. 3(a)). It is also clear that the arm excess loss (difference between insertion loss of one arm and a reference waveguide) is relatively constant until a separation of $5 \mu \mathrm{m}$ is reached, reading $8 \mathrm{~dB}$ for $15 \mu \mathrm{m}$ separation as seen in Fig. 3(b). This behavior, in the 0-5 $\mu \mathrm{m}$ zone, was not expected, as seen from the simulations, but can be explained by the Y-junction arms overlap. There is however the opportunity to optimize other features without further loss degradation using arm separation, such as the junction spectral behavior, since insertion loss is approximately constant up to $5 \mu \mathrm{m}$.
For this a spectral analysis was performed from $1300 \mathrm{~nm}$ to $1600 \mathrm{~nm}$ (Fig. 4). As can be seen in the first graphic of Fig. 4, the asymmetric index distribution discussed previously is also responsible for asymmetrical spectral arm behavior; such feature is not ideal for broadband power splitters. Similarly to Fig. 3(b), Fig. 4 shows the existence of two distinct regions; the first, ranging from $0 \mu \mathrm{m}$ to $5 \mu \mathrm{m}$, where both arms perform asymmetrically and the experimental and simulated behavior do not match; and the second, from $5 \mu \mathrm{m}$ to $15 \mu \mathrm{m}$, where both arms behave symmetrically and the experimental and simulated behavior do match. Originally, arm 1 loses more power at lower wavelengths while arm 2 shows a stable behavior. As arm separation increases the symmetry also increases until both arms match the simulated behavior (at $5 \mu \mathrm{m}$ ). This means that the writing of the second arm is no longer affecting the first, making the junction physically symmetrical for a separation distance greater than $5 \mu \mathrm{m}$. This behavior resembles the characteristics already seen in directional couplers [23], where the coupling coefficient increases for increasing wavelength, meaning that, for a small separation, everything, from $1300 \mathrm{~nm}$ to $1600 \mathrm{~nm}$, can be coupled to the output waveguides. However, as the separation increases the lower wavelengths coupling efficiency diminish, 
thus the observed increased losses. As the arm separation increases even further the longer wavelengths, with higher coupling coefficients, do not have enough interacting length to couple and also suffer increased loss. The vertical shift observed when both arms have the same behavior is likely due to misalignment of the output butt coupled fiber.

In summary, this technique appears to be suited for junction asymmetry correction, with the best results being obtained for a $5 \mu \mathrm{m}$ arm separation. The $5 \mu \mathrm{m}$ arm separation Y-junction has a power splitting of 50.9:49.1, a junction loss of $0.4 \mathrm{~dB}$ at $1550 \mathrm{~nm}$, as well as $4.5 \mathrm{~dB}$ insertion loss (including the $3 \mathrm{~dB}$ splitting loss) and $0.20 \mathrm{~dB}$ polarisation dependent loss (PDL) for a $2.5 \mathrm{~cm}$ long device (although the junction is only $6.2 \mathrm{~mm}$ long). Since the waveguide fabrication parameters were optimized towards minimizing propagation loss, most insertion loss arises from the coupling to the SMF-28 fiber and should be substantially reduced if this problem is addressed. Regarding the spectral behavior, this junction shows only a small decrease of $0.48 \mathrm{~dB}$ from $1600 \mathrm{~nm}$ to $1300 \mathrm{~nm}$, while maintaining the power splitting. Several applications can benefit from this study, with the most striking example being integrated optical chips used for beam combining in astronomical interferometry [24]. In this particular application, the optical signals are broadband $(\sim 300 \mathrm{~nm}$ wide $)$ and therefore achromatic behavior is essential. Integrated Mach-Zehnder interferometric sensors [25] can also benefit from this advance.

\section{CONCLUSION}

A new design for the fabrication of balanced broadband Y-junction power splitters, based on arm separation, with the femtosecond laser direct writing technique was tested and optimized. Subsequently, a $6.2 \mathrm{~mm}$ long Y-junction power splitter with relatively low insertion loss, good power splitting uniformity and low wavelength dependent loss was fabricated with a $5 \mu \mathrm{m}$ arm separation in a $2.5 \mathrm{~cm}$ long fused silica substrate. This enables more complex binary tree structures, for $1 \times \mathrm{N}$ power splitting, with the same characteristics to be produced. The insertion loss (including $3 \mathrm{~dB}$ splitting loss), splitting uniformity, PDL and wavelength dependent loss obtained were $4.5 \mathrm{~dB}, 0.15 \mathrm{~dB}(50.9: 49.1), 0.20 \mathrm{~dB}$, and $0.48 \mathrm{~dB}$ respectively, in the wavelength range of $1300 \mathrm{~nm}$ to $1600 \mathrm{~nm}$. The results shown in this letter have a positive impact on devices that require achromatic Y-junction splitters, such as integrated optical chips for beam combining in astronomical interferometry and sensors based on Mach-Zehnder interferometers, for example.

\section{REFERENCES}

[1] L. B. Soldano, F. B. Veerman, M. K. Smit, B. Verbeek, A. H. Dubost, and E. C. M. Pennings, "Planar monomode optical couplers based on multimode interference effects," J. Lightw. Technol., vol. 10, no. 12, pp. 1843-1850, Dec. 1992.

[2] H. Yamada, T. Chu, S. Ishida, and Y. Arakawa, "Optical directional coupler based on Si-wire waveguides," IEEE Photon. Technol. Lett., vol. 17, no. 3, pp. 585-587, Mar. 2005.

[3] Y. Wang, S. Gao, K. Wang, and E. Skafidas, "Ultra-broadband and low-loss $3 \mathrm{~dB}$ optical power splitter based on adiabatic tapered silicon waveguides," Opt. Lett., vol. 41, no. 9, pp. 2053-2056, May 2016.
[4] S. H. Tao, Q. Fang, J. F. Song, M. B. Yu, G. Q. Lo, and D. L. Kwong, "Cascade wide-angle Y-junction $1 \times 16$ optical power splitter based on silicon wire waveguides on silicon-on-insulator," Opt. Exp., vol. 16, no. 26, pp. 21456-21461, Dec. 2008.

[5] R. Dangel and W. Lukosz, "Electro-nanomechanically actuated integrated-optical interferometric intensity modulators and $2 \times 2$ space switches," Opt. Commun., vol. 156, nos. 1-3, pp. 63-76, Nov. 1998.

[6] K. Okamoto, "Beam propagation method," in Fundamentals Optical Waveguides, vol. 2, 2nd ed. Cambridge, MA, USA: Academic, 2006, ch. 7, pp. 329-383.

[7] L. Wang et al., "A compact $1 \times 64$ optical power splitter using silicabased PLC on quartz substrate," Opt. Laser Technol., vol. 61, pp. 45-49, Sep. 2014.

[8] J. Gamet and G. Pandraud, "Ultralow-loss $1 \times 8$ splitter based on field matching Y junction," IEEE Photon. Technol. Lett., vol. 16, no. 9, pp. 2060-2062, Sep. 2004.

[9] N. Bellini et al., "Femtosecond laser fabricated monolithic chip for optical trapping and stretching of single cells," Opt. Exp., vol. 18, no. 5, pp. 4679-4688, Mar. 2010.

[10] K. Faerch and M. Svalgaard, "Symmetrical waveguide devices fabricated by direct UV writing," IEEE Photon. Technol. Lett., vol. 14, no. 2, pp. 173-175, Feb. 2002.

[11] W. Yang, C. Corbari, P. G. Kazansky, K. Sakaguchi, and I. C. S. Carvalho, "Low loss photonic components in high index bismuth borate glass by femtosecond laser direct writing," Opt. Exp., vol. 16, no. 20, pp. 16215-16226, Sep. 2008.

[12] W. Yuan, J. Lv, C. Cheng, X. Hao, and F. Chen, "Waveguides and proportional beam splitter in bulk poly(methyl methacrylate) produced by direct femtosecond-laser inscription," Opt. Mater., vol. 49, pp. 110-115, Nov. 2015.

[13] R. He, I. Hernández-Palmero, C. Romero, J. R. V. de Aldana, and F. Chen, "Three-dimensional dielectric crystalline waveguide beam splitters in mid-infrared band by direct femtosecond laser writing," Opt. Exp., vol. 22, no. 25, pp. 31293-31298, Dec. 2014.

[14] J. Lv, Y. Cheng, W. Yuan, X. Hao, and F. Chen, "Threedimensional femtosecond laser fabrication of waveguides beam splitter in $\mathrm{LiNbO}_{3}$ crystal," Opt. Mater. Exp., vol. 5, no. 6, pp. 1274-1280, May 2015.

[15] J. Liu, Z. Zhang, S. Chang, C. Flueraru, and C. P. Grover, "Directly writing of 1-to-N optical waveguide power splitters in fused silica glass with femtosecond laser," Opt. Commun., vol. 253, nos. 4-6, pp. 315-319, Sep. 2005.

[16] I.-B. Sohn, M.-S. Lee, and J.-Y. Chung, "Fabrication of optical splitter and passive alignment technique with a femtosecond laser," IEEE Photon. Technol. Lett., vol. 17, no. 11, pp. 2349-2351, Nov. 2005.

[17] I. Sohn, M. Lee, H. Park, and H. Lee, "Direct writing of a 1-to-4 optical splitter and packaging technology using a femtosecond laser," J. Korean Phys. Soc., vol. 52, no. 4, pp. 1120-1122, Apr. 2008.

[18] L. A. Fernandes, J. R. Grenier, P. R. Herman, J. S. Aitchison, and P. V. S. Marques, "Femtosecond laser writing of waveguide retarders in fused silica for polarization control in optical circuits," Opt. Exp., vol. 19, no. 19, pp. 18294-18301, Sep. 2011.

[19] L. A. Fernandes, J. R. Grenier, P. R. Herman, J. S. Aitchison, and P. V. S. Marques, "Stress induced birefringence tuning in femtosecond laser fabricated waveguides in fused silica," Opt. Exp., vol. 20, no. 22, pp. 24103-24114, Oct. 2012.

[20] R. Osellame, G. Cerullo, and R. Ramponi, "Quill and nonreciprocal ultrafast laser writing," in Femtosecond Laser Micromachining: Photonic and Microfluidic Devices in Transparent Materials, vol. 6, 1st ed. New York, NY, USA: Springer, 2012, pp. 127-150.

[21] Y. Nasu, M. Kohtoku, and Y. Hibino, "Low-loss waveguides written with a femtosecond laser for flexible interconnection in a planar light-wave circuit," Opt. Lett., vol. 30, no. 7, pp. 723-725, Apr. 2005.

[22] S. M. Eaton, H. Zhang, and P. R. Herman, "Heat accumulation effects in femtosecond laser-written waveguides with variable repetition rate," Opt. Exp., vol. 13, no. 12, pp. 4708-4716, Jun. 2005.

[23] L. A. Fernandes, J. R. Grenier, P. R. Herman, J. S. Aitchison, and P. V. S. Marques, "Femtosecond laser fabrication of birefringent directional couplers as polarization beam splitters in fused silica," Opt. Exp., vol. 19, no. 13, pp. 11992-11999, Jun. 2011.

[24] J. LeBouquin et al., "Comparison of integrated optics concepts for a near-infrared multi-telescope beam combiner," Proc. SPIE, Glasgow, Scotland, vol. 5491, pp. 1362-1369, Oct. 2004.

[25] A. Crespi et al., "Three-dimensional Mach-Zehnder interferometer in a microfluidic chip for spatially-resolved label-free detectiongf," $L a b$ Chip, vol. 10, no. 9, pp. 1167-1173, May 2010. 